\title{
Bandwidth Problem in High Performance Packet Switching Network
}

\author{
Syed S. Rizvi ${ }^{1}$, Aasia Riasat ${ }^{2}$, Muhammad S. Rashid ${ }^{3}$, and Khaled M. Elleithy ${ }^{4}$ \\ Computer Science and Engineering Department, University of Bridgeport ${ }^{1,3,4}$, Bridgeport CT, 06601 \\ Department of Computer Science, Institute of Business Management ${ }^{2}$, Karachi, Pakistan \\ $\left\{\right.$ srizvi $^{1}$, muhammsi ${ }^{3}$, elleithy $\left.{ }^{4}\right\} @$ bridgeport.edu, aasia.riasat@cbm.edu.pk ${ }^{2}$
}

\begin{abstract}
High performance packet switching networks are being deployed to provide sufficient data bandwidth for end users 3G services such as video streaming and broadband like data services. The use of high performance networks is, therefore, essential to the success of any $3 G$ service. However, in practice, the deployment of High performance packet switching networks is hindered due to the improper congestion control which consequently results longer delays. In this paper, we propose a new method that can effectively improve the congestion control in high performance packet switching networks. Our numerical and simulation results demonstrate that the proposed method can be implemented for both lightly and heavily loaded networks. Simulation results show that the transmission delays can also be reduced significantly that improves the over all performance of high performance packet switching networks
\end{abstract}

Keywords - high performance networks, 3G services, congestion control, transmission delays

\section{INTRODUCTION}

As in Packet Switching Networks the message is decomposed into packets which share channels with other packets [3]. Packet high performance networking strives to achieve the highest possible latency, high utilization of the expensive resources, fair allocation of resources to competing users (QOS guarantees), or combinations of all these. Actually, Packet Switching network was originally designed to provide more efficient facility than circuit switching for bursty data traffic. Here the bandwidth seems utilized more efficiently. Main advantages of Packet Switching are flexibility, resource sharing, robustness, responsiveness.

Today's internet is built up as a giant packet switched network. Since packet switching offer better bandwidth sharing and is less costly to implement than circuit switching, it is widely used in data networks [1]. The internet transmits data packets efficiently but it provides no guarantee on the end to end transmission delay (up bound, jitter etc). Packet losses take place due to congestion [2, 3]. Moreover delay predictions are very important for the design of routing and flow control algorithm $[4,5]$.

\section{A. Problem Statement}

Congestion is a problem that occurs on shared networks when multiple users vie for access to the same resources (bandwidth, buffers and queues) [5]. In packet switched network, packets move in and out of the buffers and queues of switching devices as they traverse the network. In fact, a packet switched network is often referred to as a "network of queues". A characteristic of packet switched networks is that packet may arrive in bursts from one or more source. Buffer help routes absorb bursts until they can catch up. If traffic is excessive, buffers is not a solution because too much buffer size can lead to excessive delay.

The current analysis of congestion control algorithms shows that as the combination of both delay and the bandwidth increases, the transmission control protocol becomes oscillatory that makes the overall system slightly instable. Recently, reference [11] shows that the increase in the product of capacity and delay results instability in a random early discard [7], random early marking [6], proportional integral controller [9], and virtual queue [8]. In addition, it has been shown that an active queue management scheme can maintain stability over very high-capacity or large-delay links. Also, it has also been shown that the adaptive virtual queue [10] also becomes prone to instability when the link capacity is large enough.

Congestion occurs when various sources compete for network resources, and these resources cannot handle the demand [3]. This may happen when logical channels request bandwidth that cannot be supported, or when the network admits more packets than the links can handle, or at any node due to buffer shortage. Here we select problem called congestion so that we can make packet switch network more efficient. Congestion problem leads to packet loss so if we can minimize its effect then we can improve the performance of high performance packet switched network. 


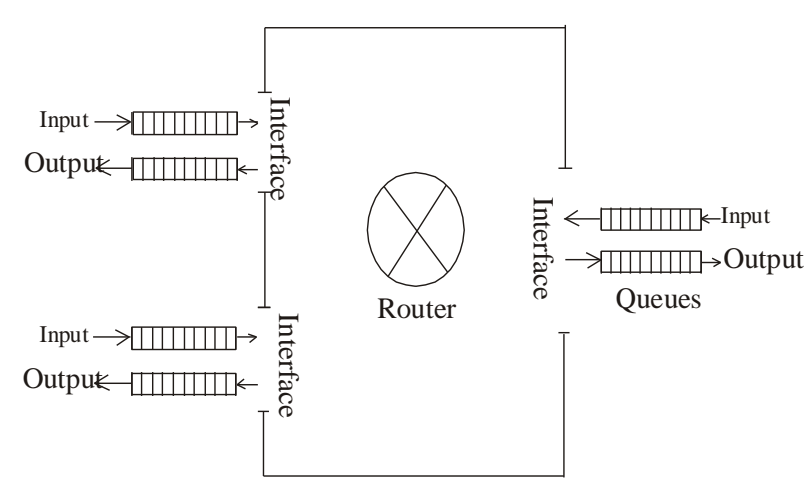

Fig. 1. Queues in the Router

Congestion in a network occurs because routers and switches have queues buffers that hold the packets before and after processing. A router for example, has an input queue and an output queue for each interface. When a packet arrives at the incoming interface .It undergoes three steps before departing.

- The packet positions at input queue while waiting to be checked.

- The processing module of the router removes the packet from queue and uses its routing table to make decision.

The packet is plant in the appropriate output queue and waits its turn to be sent.

\section{RELATED WORK}

Much research has been done to lessen the congestion problem in the network. Among them two methods to control the congestion are described under.

\section{A. Backpressure Mechanism}

The technique of backpressure refers to a congestion control mechanism in which a congested node stops receiving data from the immediate upstream node [2,7]. This may cause the upstream node to become congested, and they in turn, reject data from their upstream nodes and so on. Backpressure is a node to node congestion control that starts with a node and propagates, in the opposite direction of data flow to the source. The node 2 will have the congestion so it will inform node one to slow down. If congestion occurs in node 1 then it will inform source to slow down. This is how backpressure works.

\section{B. Choke Packet Mechanism}

A choke packet is a packet sent by a node to the source to inform it of congestion. In choke method the warning is from the router, which has encountered congestion to the source station directly $[4,11]$. The intermediate nodes through which the packet has traveled are not warned. Instead the source are warned about the congestion occurs in the router.

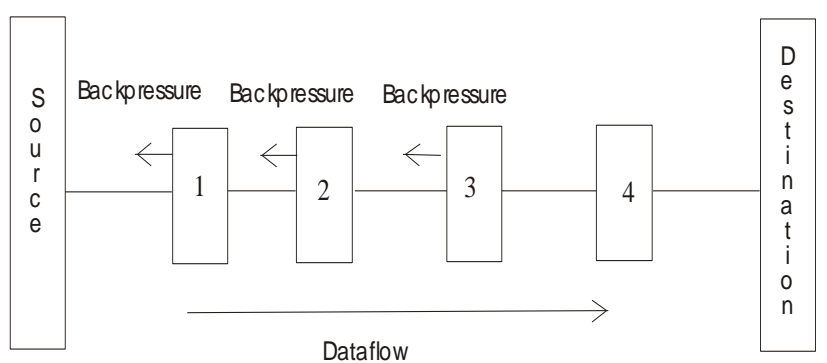

Fig. 2. Backpressure method

\section{Proposed Solution For Traffic Management In ATM NETWORKS}

Last two solutions loss the data. We have proposed the elucidation without any loss of data. Here we made some assumptions, they are as follows:

- The sender and receiver know the size of the buffer the router have.

- The packet size is known by both.

The sender and destination knows the maximum time for processing the maximum size of buffer the router has. In Fig.5 the router informs the congestion to the source rather than node. Firstly the source sends the packet to the router. As the source knows the buffer size of the router and its processing time, In addition to the packet it also sends the control bit. The control bits are sent from the router to sender in certain interval. Here, we made the assumption that if the control bits from the router send that the buffer is $70 \%$ full then the sender will stop sending the packets. If the buffer size of router is $50 \%$ full then the sender will slow down by $25 \%$. Similarly if router buffer is even less than the $50 \%$ then the sender will send the packet in full speed. After certain interval the control bits are

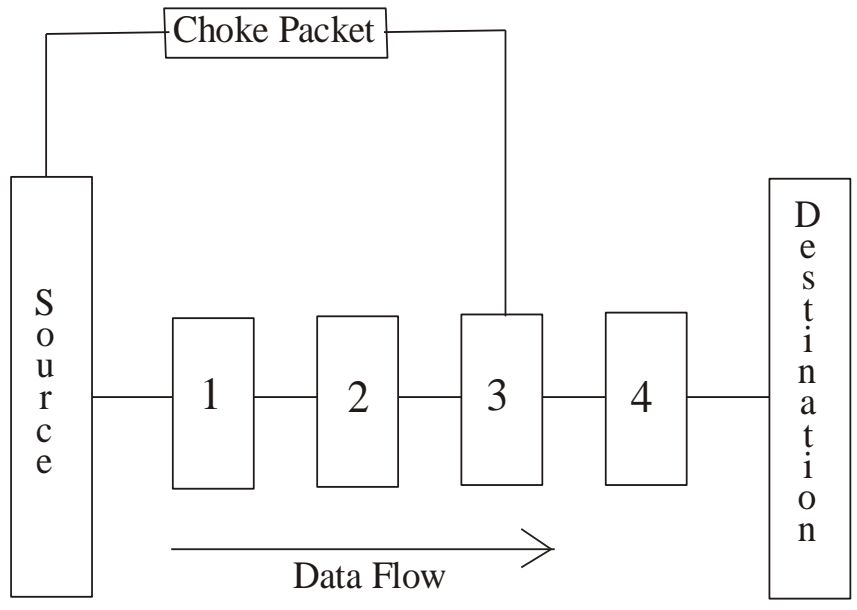

Fig. 3. Choke Packet Mechanism 
broadcasted to each sender. So by looking at the buffer usage percentage we can slow down the speed. The more it takes time to return the slower will be the packet delivery from the source. Here we take $n$ number of senders.

Now, lets move to the congestion occurs in output side of the router as shown in Fig. 6. We know the destination knows time of processing of the packet as well as the time to get that packet which is sends by the source. Suppose the destination didn't get the packet in time then the destination will send the control bit. If control bit takes time to come back to the receiver. The receiver will know there is congestion occurring in the output buffer of the router. This implies that, if router gets control bit from the receiver the router is going to slow down the processing speed so that there will be time for the receiver to get the packet without loss.

\section{Proposed Mathematical Model}

Before going to present the mathematical model, it is worth mentioning some of our assumptions:

- First, we consider both sender and router part.

- We assume that we may have $n$ number of senders

- Also, we assume that the length of the link is $L_{n}$ whereas the sender is represented by $S_{n}$

Taking this into account, we can present the following mathematical expression to formulate out proposed hypothesis as follows:

$$
\text { Total no of senders }=\sum_{n=1}^{\infty} S_{n}
$$

In the same manner, we can compute the transmission time as follows:

$$
\text { Transmission Time }=T s=L / B \quad \text { Property }(2)
$$

where $L$ is the length of the packet (typically measure in bits) and $B$ is the bandwidth of the link (typically measure in bits/sec). Combining property (1) with (2), results the following mathematical expression: In addition to that, we also

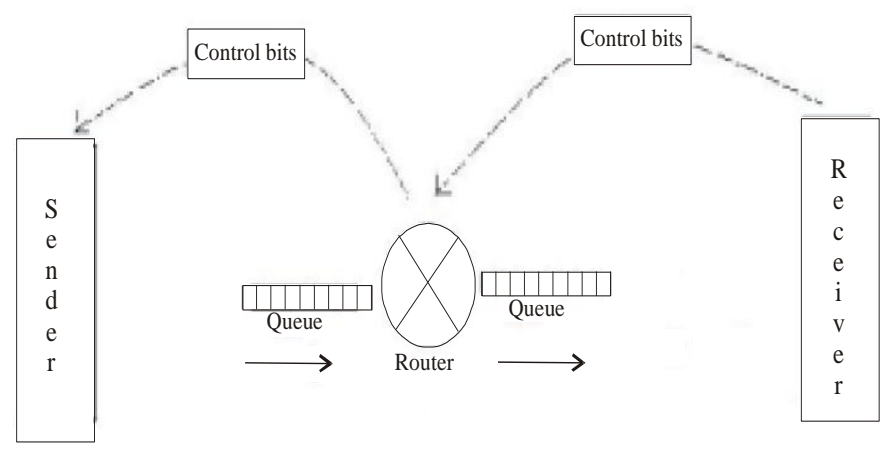

Fig. 4. Congestion control technique

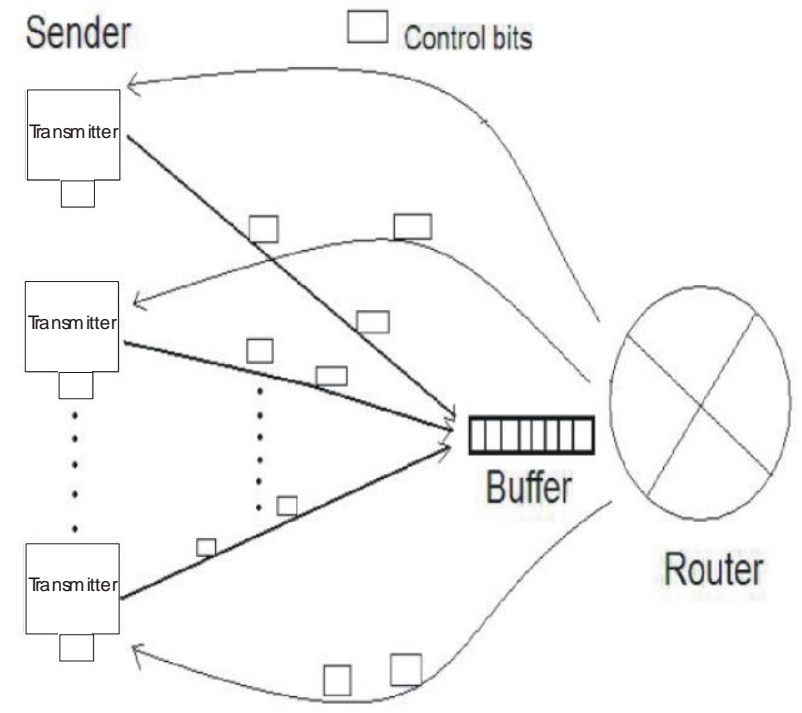

Fig. 5. Sender and Router Communication packet

assume that the propagation time remains constant for both transmission of messages and represented by $T_{p}$. With this assumption, the total time taken by $n$ number of senders to send first bit will be approximated as follows:

$$
. T_{s}(\text { total })=\sum_{n=1}^{\infty} S_{n} \times T_{n}
$$

Property (3)

$$
\text { Router }=\sum_{n=1}^{\infty}\left(T_{s}(\text { total })+T_{P}\right) S_{n}
$$

According to one of our assumptions, there are $n$ no of receivers whereas the length of the link between the router and the receiver is assumed to be $L r$. It should also be noted that the same link for the receiver side is used and represented by DSI. Taking these factors into account and combine them with the four properties presented above, we can present the following mathematical expression for the total number of receivers.

$$
\text { Total no of receiver }=\sum_{n=1}^{\infty} R n
$$

Property (5)

Also, we assume that the Processing-Time $\left(P_{r}\right)$ that router takes is $R t$. In addition, this time is assumed to be known by both sender and the receiver. This implies that the time required to transmit a single bit is a sum of processing time and the router processing time. This can be mathematically expressed as:

$$
\text { Trnamission-Time } / \text { bit }=P_{r}+R_{t}
$$

Property (6)

This can be generalized for $n$ number of receivers 

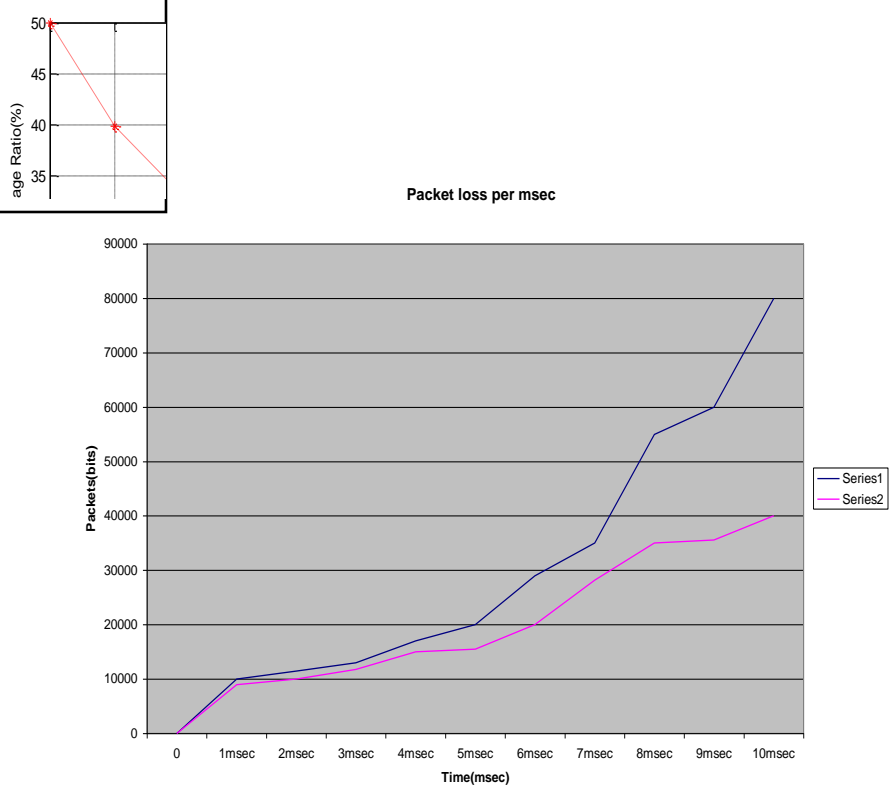

Fig.6. packet loss versus time (msec)

$$
R_{n}=\sum_{n=1}^{\infty}\left(P_{r}+R_{t}\right) R_{n}
$$

$$
\text { where } R_{n} \stackrel{\text { approximate }}{\longrightarrow} n \# \text { of } \operatorname{Re} \text { ceivers }
$$

\section{Simulation Results of the Proposed Scheme}

Fig. 6 represents an approximation of packet loss with respect to time. As harmony with Fig. 6, an average packet loss of the presented model can be numerically approximated using above properties:

$$
(T s)=1000 / 1544=0.647 \mathrm{sec} .
$$

On the other hand, average packet loss of previous model is typically presented as: Ploss $($ old $)=30045.45$. This further leads us to the following performance analysis.

$$
\text { performance }=\frac{\text { Ploss }(\text { old })-\text { Ploss }(\text { present })}{\operatorname{Ploss}(\text { old }) * 100} \square 33.4 \%
$$

where $\square$ represents estimated value

Here, we can take out the transmission time, we are using DS1 link in between the router and the sender. So the bandwidth is $1.544 \mathrm{Mb} / \mathrm{sec}$ and frame or packet size to be 1000kbits.

\section{Conclusion}

There are lots of things to be considered for the control of congestion problem. Either you have to compromise with the time, cost or packet loss. In our model, we have compromised with the time and cost but put effort to minimize the packet loss. As we all know that packet switching network is based upon connectionless network. Due to this there is lots of packet loss. According to the previous model we found loss of data to be maximum. So we somewhat tried to diminish the packet loss by making some of the assumptions.

\section{REFERENCES}

[1] L. Klein, S. Lam, "Packet Switching in a Multi-access Broadcast Channel: Performance Evaluation," IEEE Transaction on Communications, Vol. 23, Issue. 4, pp. 410- 423.

[2] S. Kalyanasundaram, J. Li, E.K.P. Chong and N.B. Shroff, Channel sharing scheme for packet-switched cellular networks, in: IEEE INFOCOM'99 New York (March 1999) pp. 609-616.

[3] B. Vandalore, W. Feng, R. Jain, S. Fahmy: A Survey of Application Layer Techniques for Adaptive Streaming of Multimedia. Real-Time Imaging 7(3): 221-235 (2001)

[4] H. Jiang and S. Rappaport, CBWL: A new channel assignment and sharing method for cellular communication systems, IEEE Transactions on Vehicular Technology, Vol. 43, Issue. 2, pp. 313-322, 1994.

[5] R. Jain, K. Ramakrishna, D. Chiu, "Congestion Avoidance in Computer Networks with a Connectionless Network Layer," Innovation in Internetworking, pp. 140 - 156, 1998.

[6] S. Athuraliya, V. Li, S. Low, and Q. Yin, "Active queue management," IEEE Network, 2001.

[7] S. Floyd and V. Jacobson, "Random early detection gateways for congestion avoidance," In IEEE/ACM Transactions on Networking, Vol. 1, Issue. 4, pp. $397-$ 413, Aug. 1993

[8] R. Gibbens and F. Kelly, "Distributed connection acceptance control for a connectionless network," In Proc. of the $16^{\text {th }}$ Intl. Telegraffic Congress, June 1999

[9] C. Hollot, V. Misra, D. Towsley, and W. Gong, "On designing improved controllers for AQM routers supporting TCP flows," In Proc. of IEEE INFOCOM, Apr. 2001

[10] S. Kunniyur and R. Srikant, "Analysis and design of an adaptive virtual queue," In Proc. of ACM SIGCOMM, 2001.

[11] S. Low, F. Paganini, J. Wang, S. Adlakha, and J. Doyle, "Dynamics of TCP/AQM and a scalable control," In Proc. Of IEEE INFOCOM, June 2002. 